\title{
CIENCIA DE PAPEL. PRIVILEGIOS Y AUTORIDAD EN ESPAÑA DURANTE EL PRIMER FRANQUISMO (1939-1959)
}

\author{
SCIENCE MADE OF PAPER. PRIVILEGES AND AUTHORITY \\ IN FRANCO'S SPAIN (1939-1959)
}

Fernando García Naharro*

Europa-Universität Flensburg - Alemania

\begin{abstract}
RESUMEN: Este artículo pretende analizar la lógica que gobierna las prácticas editoriales en el ámbito científico y técnico de la España franquista. Para ello, se atiende tanto a la estructura subyacente al campo editorial como al proceso mismo de confección de las publicaciones del Consejo Superior de Investigaciones Científicas (CSIC), la principal institución científica de aquel tiempo. Así, se aborda el estudio de la construcción la autoridad científica en la España franquista a través de las publicaciones, puesta en estrecha relación con el nombre y la figura del autor, así como con el nombre y el prestigio de la editorial que se decide a realizar su publicación impresa. Publicaciones cientificas y técnicas que, en última instancia, estarán condicionadas no sólo por el orden del discurso bajo un régimen dictatorial, sino también por las vicisitudes propias de su proceso de producción.
\end{abstract}

PALABRAS CLAVE: Consejo Superior de Investigaciones Científicas, Ciencia, Franquismo, Publicaciones científicas, España.

ABSTRACT: This paper aims to study the publishing practices regarding to scientific and technical literature in Franco's Spain. In so doing, I proceed exploring the structure of the Spanish publishing field and the publishing process of scientific and technical literature linked to the Spanish National Research Council (CSIC), the major Spanish scientific institution under Franco. With this, I study the shaping of the scientific authority in Franco's Spain through the publications, which are defined in relation to the author function and the prestige of those publishing services involved. Framed by this premise, the scientific and technical literature is conditioned by the order of discourse under a dictatorial regime, but also by the publishing process itself.

KEYWORDS: Spanish National Research Council, Science, Franco's dictatorship, Scientific and Technical Literature, Spain.

* Correspondencia a / Corresponding author: Fernando García Naharro. Romanisches Seminar. Institut für Sprache, Literatur und Medien, Europa-Universität Flensburg, Auf dem Campus 1, 24943, Flensburg (Alemania) - fernando.garcianaharro@uni-flensburg.de - https://orcid.org/0000-0002-1412-4659

Cómo citar / How to cite: García Naharro, Fernando (2022). "Ciencia de papel. Privilegios y autoridad en España durante el primer franquismo (1939-1959)", Historia Contemporánea, 68, 233-262. (https://doi.org/10.1387/hc.21564).

Recibido: 17 marzo, 2020; aceptado: 27 julio, 2020.

ISSN 1130-2402 - eISSN 2340-0277 / (C) 2022 Historia Contemporánea (UPV/EHU) 


\title{
1. Objetos impresos: las publicaciones científicas del CSIC en la España franquista
}

\begin{abstract}
El problema es claro (...) hay que preguntar si la investigación científica puede seguir interrumpida, sus publicaciones suspensas, su intercambio sin reanudar, mientras los rojos emigrados comienzan a publicar y los que aquí han quedado piensan que sin los que se han ido no somos capaces de organizar nada ${ }^{1}$
\end{abstract}

Planificar la investigación nacional en plena posguerra española no era tarea fácil. Así lo reflejan estas palabras escritas, en abril de 1940, por el propio José María Albareda (1902-1966)², Secretario General del Consejo Superior de Investigaciones Científicas (CSIC) ${ }^{3}$, la flamante institución científica nacional que el régimen franquista fundó, el 24 de noviembre de 1939, sobre los escombros de la Junta para Ampliación de Estudios e Investigaciones Científicas (JAE) ${ }^{4}$. No en vano, la irrupción del CSIC en el campo científico español truncó abruptamente la encomiable labor realizada por la JAE, decretándose tanto el cese oficial de sus actividades durante la Guerra Civil como su posterior disolución en 1939, momento en el que sus centros quedaron ligados al CSIC. Desde entonces, el Consejo pasó a ser el principal organismo de investigación de España y el órgano rector de la política científica española; así sería, al menos, hasta finales de los cincuenta cuando, ante la inoperancia del modelo de coordinación de la ciencia española, entre en escena la Comisión Asesora de Investigación Científica y Técnica (1958), la primera de una serie de iniciativas estatales para dotar a España de una política científica coherente ${ }^{5}$.

«La realidad es que se puede organizar una investigación nacional con unas dimensiones y una hondura muy superiores a todo lo pasado - continuaba relatando, en 1940, José María Albareda - Treinta años de Junta para ampliación de estudios nos han dejado ramas fundamentales sin intento de labor alguna: en Filosofía, Geografía, Técnica ni siquiera se

${ }^{1}$ Carta de José María Albareda a José Lorente (Subsecretario de Gobernación) (29 de abril de 1940) (AGUN/JMA/006/001/0011).

2 Malet, 2009; Gutierrez Ríos, 1970.

3 Santesmases; Muñoz, 1993; Sánchez Ron, 1998; Canales, 2015.

4 Sánchez Ron, 1988; Puig-Samper Mulero, 2007; Caballero Garrido, 2010; Otero Carvajal; López Sánchez, 2012.

5 Nieto, 1982; Delgado; López, 2019. 
pensó» ${ }^{6}$. Urgía entonces poner remedio, enderezar el rumbo y «reparar el injustificable olvido» que suponía, por ejemplo, el que - según palabras del presidente del CSIC y Ministro de Educación Nacional, José Ibáñez Martín (1896-1969) - la investigación técnica y aplicada no se hubiera cultivado en España hasta la creación del Consejo ${ }^{8}$. Presuntos olvidos que justificarían, a su vez, una visible variación en las líneas y los ámbitos de investigación: caso, por ejemplo, del impulso de la óptica (que «retoñaba ayer entre nosotros para ser ya hoy rama fundamental del árbol de nuestra ciencia contemporánea ${ }^{9}$ ) y la física nuclear, todo ello en consonancia con los intereses industriales y militares de la dictadura franquista ${ }^{10}$.

«No es sólo el cambio radical en la orientación de las ideas directrices del pensamiento - afirmaba José Ibáñez Martín - es la realización misma de la investigación lo que se rectifica» ${ }^{11}$. Variaciones y cambios con ínfulas de redención que, unidos a la purga de científicos contrarios al régimen y al reclutamiento de nuevos expertos legitimados ${ }^{12}$, dibujaron los contornos de la ciencia oficial de posguerra: una ciencia netamente nacional, pretendidamente unitaria, de raigambre católica y (supuestamente) libre $^{13}$ aunque estuviera, a la postre, subordinada siempre a «las realidades vivas que tiene planteadas el Estado» ${ }^{14}$. Ese «Nuevo Estado» franquista que, si bien encontró en la virilidad un rasgo intrínseco de la nueva España ${ }^{15}$, otorgó, a su vez, al sujeto-varón-conocedor (ese otro modelo de hombre, el vir modestus ${ }^{16}$ ) un lugar privilegiado en el campo científico español donde, sin embargo, también algunas mujeres supieron encontrar su sitio $^{17}$. Nombres de mujeres ${ }^{18} \mathrm{y}$, mayoritariamente, hombres con limpieza

${ }^{6}$ Carta de José María Albareda a José Lorente (Subsecretario de Gobernación) (29 de abril de 1940) (AGUN/JMA/006/001/0011).

7 Presidente del CSIC (1939-1967) y Ministro de Educación Nacional (1939-1951). Datos biográficos en José Ibáñez Martín (AGA 31/11.589).

8 CSIC, 1948, p. 147.

9 CSIC, 1948, p. 146.

${ }^{10}$ Herran; Roqué, 2012, pp. 94-97.

11 CSIC, 1943, p. 52.

12 García Naharro, 2022.

13 González Blasco, 1980, pp. 145-151.

14 CSIC (1942): 2.

15 Box, 2019.

16 Haraway, 1996.

17 Santesmases, 2000; Romero de Pablos, 2017; Santesmases, 2017; Pimentel, 2020, pp. 291-342.

18 García Naharro, 2021a. 
de sangre y afines a la causa que pregonaba el franquismo: la producción de una ciencia ideológicamente correcta ${ }^{19}$. Una ciencia nacional-católica imbuida de toda una retórica autárquica de la más pura solera nacional ${ }^{20}$ que, sin embargo, no siempre casará bien con las prácticas científicas desplegadas después en los centros de investigación ${ }^{21}$.

No obstante, aquellos sabios modestos y útiles (Menéndez y Pelayo) que conformaban el CSIC ${ }^{22}$ sirvieron también de modelo al que se opondría, entonces, su «exterior constitutivo» ${ }^{23}$ : el colectivo de los científicos identificados con la República, «los hierofantes de la impiedad y de la antipatria $»^{24}$, esa «intelectualidad descastada ${ }^{25}$ y culpable de la anti-España ${ }^{26}$ que tan presente estuvo en el discurso oficial del franquismo durante el primer lustro de la década de los cuarenta. Sin embargo, todos estos argumentos desplegados a nivel discursivo tuvieron, a su vez, implicaciones prácticas en la conformación del campo científico español de la época. No en vano, hacia 1940 cerca de la mitad de los 580 profesores universitarios en activo antes de la Guerra Civil habían muerto, habían sido purgados o se habían exiliado del país ${ }^{27}$, lo que suponía una pérdida en proporción mayor que la registrada en países como Alemania, Portugal o Italia ${ }^{28}$. Así se entiende también que, a la altura de 1955, de los investigadores que trabajaban en el CSIC sólo el 5\% había publicado antes de $1940^{29}$.

Sin embargo, no por ello se dejó de publicar, siendo el Consejo Superior de Investigaciones Científicas, para el ámbito científico español, el principal órgano editor ${ }^{30}$. «Leed los sumarios de las revistas, - afirmaba, a mediados de los años cuarenta, José Ibáñez Martín - revisad los libros, mirad los concursos de premios, y advertiréis el crescendo suave y arrollador de una producción científica en marcha, de una juventud que tra-

${ }_{19}$ Para su aplicación en estudios sobre la Unión Soviética, Japón o China véase Gordin; Grunden; Walker; Wang, 2003.

20 Canales, 2019.

21 Santesmases, 2012.

22 CSIC, 1951, pp. 67-68.

23 Butler, 1993, p. 3.

24 CSIC, 1942, p. 30.

25 CSIC, 1943, p. 51.

26 CSIC, 1944, p. 48.

27 Otero Carvajal, 2006; Otero Carvajal, 2014; Claret Miranda, 2006.

28 Herran; Roqué, 2013, p. 209.

29 González Blasco; Jiménez Blanco, 1979, p. 131.

30 Instituto Nacional de Estadística, 1958, pp. 30-40 y 9-13. 
baja y cree» ${ }^{31}$. No en vano, desde 1940, el CSIC impulsó la publicación de libros y revistas científicas mediante el incremento de sus partidas presupuestarias, que pasaron de las 385.000 pesetas de 1943 a los 17, 4 millones de pesetas en 1960. Así, en 1964, el CSIC había publicado ya cerca de 3.500 títulos de monografías científicas y de humanidades, contando con más de 160 revistas especializadas dependientes de los diferentes institutos que conformaban el Consejo ${ }^{32}$. Una potente labor editorial y un abultado número de revistas con las que, al tiempo que se daban facilidades de publicación a los investigadores del CSIC, se pretendía proyectar también una imagen de ciencia vigorosa e influyente ${ }^{33}$.

Una influencia que, sin embargo, en la práctica no fue tal y que, en su apuesta por la cantidad (y en ocasiones en detrimento de la calidad), motivó la aparición de duplicidades a las que harían alusión, incluso, personalidades tan señaladas como Joaquín Ruiz Giménez (1913-2009) (Ministro de Educación) ${ }^{34}$ o el propio Gregorio Marañón (1887-1960) (Presidente de la Comisión de Publicaciones Científicas del CSIC), quien consideraba conveniente agrupar a los «lectores de las revistas que tienen un parecido sentido científico» con el fin de evitar «la asfixia del pensamiento científico, producida por su propia fecundidad, no bien controlada ${ }^{35}$. Todo un sistema endogámico de revistas y monografías publicadas por el CSIC conformadas por trabajos escritos mayoritariamente en castellano que, si bien funcionó como instrumento activo en la conformación y mantenimiento de la comunidad científica española, también lo hizo como vehículo de «revelación al exterior» de la pretendida «consagración y vitalidad interior» del Consejo ${ }^{36}$.

«Las publicaciones enviadas y recibidas en la relación con otros países, es una muestra patente - escribía José María Albareda- de esta comunicación con el mundo» ${ }^{37}$. Quizá por ello, a esta tarea habría que

31 CSIC, 1945, p. 57.

32 Malet, 2008, pp. 241-242.

33 López Sánchez; Fernández Gallego, 2021.

34 «En el orden, por ejemplo, de las publicaciones, hemos de preferir siempre la calidad a la cantidad; reajustar, en la medida en que no dañe al propio trabajo científico, el número de revistas; fomentar la concisión; restringir lo que sea materia de una curiosidad histórica o de actividad ensayista, o de generalizaciones divulgadas» (CSIC, 1952, pp. 90-91).

${ }^{35}$ Carta de Gregorio Marañón a José María Albareda (20 de julio de 1956) (AGUN/ JIM/586/A-3 (1956).

36 CSIC, 1943, p. 47.

37 Albareda, 1964, p. 8 
sumar también el trabajo de los servicios bibliográficos del CSIC articulados en torno a su Biblioteca General, cuyos fondos, en 1954, ascenderían a «más de los 100.000 ejemplares y cerca de 150.000 colecciones de revistas» ${ }^{38}$. Fondos nutridos tanto de compras de bibliotecas particulares como de incautación de bibliotecas o de fondos de la extinta $\mathrm{JAE}^{39}$, pero también, y en buena medida, de los intercambios con otras instituciones culturales. Donaciones de fondos ${ }^{40}$ y canje de libros y revistas con organismos científicos extranjeros que, unidos al envío de información bibliográfica $^{41}$, a las publicaciones ${ }^{42}$ y las estancias de investigadores españoles en el extranjero ${ }^{43}$ o a la invitación de profesores extranjeros al Consejo ${ }^{44}$, eran defendidos por Albareda como el medio propicio para dar a «conocer a nuestro personal científico y establecer esta necesaria comunidad de actividades y relaciones» con las que, afirmaba en 1964, «en algunos casos (...) las fecundas relaciones humanas hayan pasado a ser relaciones de instituciones científicas» ${ }^{45}$.

Todo ello contribuyó, por tanto, a reforzar las precarias relaciones científico-técnicas trasnacionales del régimen ${ }^{46}$, entabladas no sólo a través de acuerdos sino también mediante la circulación de conocimientos y actores sociales ${ }^{47}$. Ejemplos que nos muestran también cómo hasta los propios objetos incorporan significados: ya fuera el primer microscopio electrónico del Instituto «Daza de Valdés» de Óptica (el primero en instalarse en España (1947) $)^{48}$ o las primeras patentes de invención registradas en el Patronato «Juan de la Cierva» en 1948, estas últimas, objetos impresos utilizados retóricamente no sólo para ensalzar su interés económico, sino también como garantes de la pretendida unión productiva de todas

38 Díaz-Pines y Fernández Pacheco, 1959.

${ }^{39}$ García Naharro, 2015a,pp. 769-771.

40 Romero de Pablos, 2018.

41 García Naharro 2020a.

42 García Naharro 2020b.

43 Delgado Gómez-Escalonilla, 2007, pp. 269-278.

44 Presas i Puig, 1998, p. 346; p. 349; Formentin Ibáñez; Carrascosa; Rodriguez Fraile, 2015, pp. 99-105.

45 Albareda, 1964, p. 8.

46 Presas i Puig, 2000; Santesmases, 2007, pp. 220-230.

47 Nieto-Galan, 2019, pp. 155-163; Camprubí, 2014, pp. 137-158.

48 Que, según Ibáñez Martín, junto al valor práctico como instrumento de trabajo adquiría «otro más alto significado: viene a ser índice del generoso esfuerzo, no exento de sacrificio, con que se dota a la investigación española en tiempos difíciles» (CSIC, 1948, p. 151). 
las ciencias ${ }^{49}$. Del mismo modo, las propias publicaciones del CSIC se recubrían entonces de toda una carga simbólica al ser ofrecidas a personalidades como el Papa Pio XII ${ }^{50} \mathrm{o}$ al entregárselas anualmente al Caudillo - como «ofrenda» y testimonio impreso del crecimiento científico de España ${ }^{51}$ - en volúmenes «lujosamente encuadernados» ${ }^{52}$. Todo ello, también, parte integrante del ritual y las prácticas propias del férreo contrato social de la ciencia ${ }^{53}$ entablado entre el CSIC y el Estado; ese mediante el cual el régimen financiaba las investigaciones y aportaba los cauces para el desarrollo de la carrera científica y los canales oficiales de publicación, promoción y distribución de sus resultados ${ }^{54}$.

Dando voz a unos y excluyendo a otros, el régimen contribuyó a estructurar el campo científico, pero también a hacerlo en el ámbito de la edición científica en la España franquista: partiendo de las condiciones de flagrante desigualdad y de competencia a veces ilegal - y las más de las veces abusiva - que ejercían las entidades oficiales (Ministerios, Consejo Superior de Investigaciones Científicas, Editora Nacional, etc.) sobre la iniciativa privada de los editores comerciales, durante el franquismo se instituyó en el campo editorial español una subordinación estructural del ámbito comercial al ámbito oficial ${ }^{55}$. Una realidad sustentada no sólo a través de las desigualdades en el plano normativo y mercantil, sino también a través de la vinculación de determinados nombres, productos editoriales y servicios de publicaciones a instancias de consagración científicas en estrecha relación con la esfera política. Nombres, instancias e instituciones que, como veremos a continuación, ejercerán en España una influencia directa sobre el campo científico y el campo editorial a través de los «beneficios materiales o simbólicos que están capacitados para repartir» ${ }^{56}$.

Por todo ello, y partiendo de unos presupuestos teórico-metodológicos que nos mueven a tomar en consideración «los motivos e interacciones hu-

49 CSIC, 1950, pp. 39-40.

50 CSIC, 1944, pp. 57-64.

51 «Un exuberante conjunto de obras que atestiguan con toda rotundidad el alcance e importancia creciente de nuestra empresa» (CSIC, 1946, p. 2.).

52 CSIC, 1944, p. 53.

53 Sobre las distintas teorizaciones del contrato social de la ciencia véase Guston, 2006; Jasanoff, 2004.

${ }^{54}$ García Naharro, 2015a.

55 García Naharro, 2015b.

56 Bourdieu, 1995, p. 82. 
manas que los textos llevan aparejados ${ }^{57}$, en las siguientes páginas cederemos el protagonismo a los productos impresos de la ciencia, muchas veces sus grandes olvidados. En este caso, libros y monografías científicas del CSIC $^{58}$ que, si bien percibimos como productos finales, no debemos olvidar que en origen fueron tan sólo proyectos que, más adelante, darían forma a un libro dentro de una colección determinada o un manual científico o técnico. Proyectos que tuvieron, por tanto, la potencialidad de convertirse en objetos $^{59}$ : en nuestro caso, en productos impresos a los que ahora, sin embargo, pretendemos volver a ver como textos con los que poder reconstruir parte de los procesos que los llevaron a ser lo que finalmente fueron.

Y es que «un libro jamás llega al lector sin marcas» ${ }^{60}$ : marcas que son sistemas de clasificación implícitos y que son siempre - e irremediablemente- históricas. Por tanto, atendiendo a su materialidad pero también al valor de estos libros como artefactos en circulación y al modo en que estos productos de la ciencia adquieren valor y crédito ${ }^{61}$, en las próximas páginas trataremos de devolver a lo impreso su capacidad de agencia. No obstante, coincidiendo con Marcel Boll (1886-1971) en que los contenidos científicos son "peligrosos para los imprudentes que se arriesgan a entrar en (ellos) con armas insuficientes» ${ }^{62}$, nuestra aproximación buscará, en cambio, articular su relato en torno a los proyectos editoriales, los soportes materiales impresos y los espacios por los que circulan estos contenidos científicos y técnicos en la España franquista. Todo ello, además, situado ${ }^{63}$ en el intersticio del campo científico, el campo político y el campo editorial de la ciencia en aquella España autoritaria, jerárquica y paternalista del primer franquismo.

\section{Economía de nombres: construyendo capital simbólico}

Si para un investigador el publicar su trabajo bajo el sello del CSIC suponía un espaldarazo a su estatus como científico en la España franquista, no lo era menos para una editorial comercial: así lo podemos comprobar

\footnotetext{
57 Mckenzie, 1971, p. 32.

58 Para un análisis de las revistas del CSIC véase García Naharro, 2021b.

59 Latour, 1996, pp. 24-25.

${ }^{60}$ Bourdieu; Chartier, 2010, p. 268.

61 Johns, 1998; Secord, 2000; Frasca-Espada; Jardine 2000; Biagioli; Galison 2003.

62 Bouveresse, 2001, p. 47.

${ }^{63}$ Bourdieu, 2012.
} 
con la obra premiada (premio Francisco Franco 1945) y editada por el Patronato «Juan de la Cierva», Cálculo de estructuras reticulares, del Ingeniero de Caminos y Vocal del Pleno del CSIC, Miguel García Ortega.

No es necesario manifestar a Vd. que la confianza que dispensa a nuestra Editorial encomendándonos la impresión de la obra - escribía el gerente de la editorial Dossat, el 9 de mayo de 1947- la entenderíamos en todo su valor, vigilando, con todo interés y atención, el emplanado, tipografía, confección de gráficos, etc. etc.; tareas necesarias para una buena presentación de un libro que, como el ofrecido, merece un cuidado que, modestamente, creemos poner en todas nuestras publicaciones técnicas y que las revistas profesionales reconocen y patentizan en forma que nos llena de orgullo y satisfacción» ${ }^{64}$.

Con un total de 1.200 ejemplares (200 encuadernados en «tela sajona azul claro» por el encuadernador madrileño Baldomero Merchante (c/Bailén, 6 y Yeseros, 6)), la composición, ajuste y tirada de esta obra corrió a cargo de la imprenta de la Editorial Dossat (Plaza de Santa Ana, n. ${ }^{\circ} 9$. Madrid). Sin contar con talleres gráficos propios hasta $1958^{65}$, el Jefe de Publicaciones del CSIC le confió la impresión de la obra a la Editorial Dossat, especializada desde 1943 en contenidos científicos y técnicos. Para esta casa, el moverse en el ámbito de la ciencia institucional suponía adquirir un capital simbólico que, consideraban, repercutiría positivamente en la imagen que tenía la editorial en el ámbito profesional, científico y técnico español del momento.

Para ello, en el escrito enviado al CSIC señalaban su pretensión de que el nombre de la editorial figurase junto al del Consejo en la portada, consignando «el nombre del Consejo de Investigaciones Científicas en el lugar o lugares que la Junta de Publicaciones determinase y al pié figuraría el de Editorial Dossat, S.A., Madrid, de la misma forma que lo efectuamos con algunas publicaciones de la Escuela Especial de Ingenieros de Caminos e Industriales, como puede observar en la Elasticidad del Prof. Torroja». Nombres propios y de instituciones inscritos en obras impresas que, como en este caso, la editorial pretendía distribuir «con carácter exclusivo (...) tanto para España como para el extranjero, si bien el Consejo de Investiga-

\footnotetext{
${ }^{64}$ Carta de Editorial Dossat al Jefe de Publicaciones del Consejo Superior de Investigaciones Científicas (9 de mayo de 1947) (Archivo Editorial CSIC. Caja 7, Expediente 562)

65 García Naharro, 2017, pp. 235-245.
} 
ciones queda en plena libertad para facilitar los ejemplares que desea a entidades oficiales y particulares y los necesarios para su política de intercambios, reteniendo de la edición el número que considere conveniente» ${ }^{66}$.

Una obra, la de García Ortega, en la que al final no quedaría rastro alguno de las señas de la casa Dossat, como tampoco del prólogo apalabrado con el Jefe de Publicaciones del CSIC, Rafael de Balbín (19101978), y del que se debían de haber hecho cargo Torroja, Peña y/o Terradas ${ }^{67}$. En cambio, la obra sí contó con toda una «diversidad de fórmulas que - tal y como escribían, en agosto de 1947, desde la Editorial Dossat - requieren, por su constante repetición y especial modalidad, se proceda a la tirada inmediata de los pliegos, una vez compuestos, a fin de poder continuar la composición ${ }^{68}$. Sin embargo, la falta de papel -entregado, finalmente, en noviembre de 1948 - y la laboriosa confección de los matrices especiales que requería el original retrasaron la salida de una obra que, en enero de 1949, estaba aún falta de encuadernación y de definir la nomenclatura que debían asignar al Patronato ${ }^{69}$, «el color que debe darse a la portada, que irá a dos tintas, y si debe ponerse - preguntaban desde la casa Dossat - al dorso o contratapa el escudo del Consejo, el Árbol de la Ciencia». Un emblema que, tal y como informaban desde el CSIC, debía «figurar siempre en la segunda tapa - página exterior- de todas las publicaciones de este Organismo» ${ }^{70}$.

Decía Pierre Bourdieu (1930-2002) -y, francamente, no se equivocaba - que habría que realizar una sociología comparada de los prefacios, pues «son actos típicos de transferencia de capital simbólico» ${ }^{71}$. En ese sentido, y en los siguientes términos, escribían, en octubre de 1956, desde la

66 Carta de Editorial Dossat al Jefe de Publicaciones del Consejo Superior de Investigaciones Científicas (9 de mayo de 1947) (Archivo Editorial CSIC. Caja 7, Expediente 562)

${ }^{67}$ Carta de Carta de Editorial Dossat al Jefe de la Sección de Publicaciones del CSIC (10 agosto 1948) (Archivo Editorial CSIC. Caja 7, Expediente 562)

68 Carta de Editorial Dossat al Jefe de la Sección de Publicaciones del CSIC (28 de agosto de 1947)(Archivo Editorial CSIC. Caja 7, Expediente 562)

69 «Por cierto que nos advierte el Sr. García Ortega que el título del Patronato que debe figurar en la portada es: «PATRONATO JUAN DE LA CIERVA DE INVESTIGACIÓN TÉCNICA» y no «PATRONATO JUAN DE LA CIERVA CODORNIU» que reza en el fotograbado remitido» (Carta de Editorial Dossat al Jefe de la Sección de Publicaciones del CSIC (7 de enero de 1949)(Archivo Editorial CSIC. Caja 7, Expediente 562). Finalmente se publicó sin la modificación sobre la nueva nomenclatura del Patronato.

70 Carta del Jefe de la Sección de Publicaciones del CSIC a la Editorial Dossat (10 de enero de 1949) (Archivo Editorial CSIC. Caja 7, Expediente 562)

71 Bourdieu, 2012, p. 172. 
editorial Gustavo Gili al director del Instituto Técnico de la Construcción y del Cemento del CSIC, Eduardo Torroja (1899-1961): «sería para nosotros un verdadero honor poder enriquecer nuestra edición española, de la que paralelamente se publicarán en el extranjero otras coediciones, con un texto firmado por usted». Enterados como estaban en la señera editorial barcelonesa ${ }^{72} \mathrm{de}$ que, recientemente, Torroja había impartido una conferencia sobre el ingeniero y arquitecto italiano Pier Luigi Nervi (1891-1979) — autor del que la editorial iba a publicar una traducción, con prólogo de Ernesto N. Rogers (1909-1969) y del propio Nervi-, se preguntaban si ellos podrían incluir dicha ponencia, con alguna modificación, para así «avalorar nuestra edición española, dada la admiración que por lo visto siente por Nervi y el merecido prestigio que goza usted, no sólo en nuestro país sino incluso en el extranjero, especialmente entre el público dedicado a la arquitectura y la construcción, que constituyen precisamente los lectores de esta obra ${ }^{73}$. Lamentablemente, en esta ocasión, la carta no encontraría contestación por parte del ilustre ingeniero del CSIC, ese organismo científico nacional que daba cobijo a toda una serie de investigadores que serían, además, como veremos, los protagonistas de su producción impresa.

\subsection{Capital simbólico: premios como elemento diferenciador}

Esos nombres de científicos españoles certificados por la institución y legitimados por el régimen serán los que, salvo excepciones, ocupen las cubiertas de las monografías publicadas por el Consejo; muchas de ellas, además, testimonio impreso de iniciativas oficiales como los premios Francisco Franco $^{74}$ (de 50.000 pesetas) o los premios Juan de la Cierva al estímulo de la investigación técnica (de 40.000 pesetas y de 20.000 pesetas) entre otros ${ }^{75}$. Generalmente, entre sus autores figurarán nombres

72 Gili; Puente; Rojals, 2013.

${ }^{73}$ Carta de editorial Gustavo Gili a Eduardo Torroja (3 de octubre de 1956) (BC, Fons Editorial Gustavo Gili, Capsa 170, MS 9500/6, carpeta TORROJA, Eduardo)

74 «Premios, en los que va no sólo el nombre, sino la viva expresión del afectivo Patronato que el Caudillo ejerce sobre toda la obra del Consejo» (CSIC, 1942, p. 9)

75 Otros estímulos del Consejo serían los premios Raimundo Lulio, Antonio de Nebrija y Luis Vives para las disciplinas de Letras o los premios Alfonso el Sabio, Santiago Ramón y Cajal o Alonso de Herrera para las de Ciencias (de 20.000 pesetas cada uno), también los premios Menéndez y Pelayo o Leonardo Torres Quevedo (de 5.000 pesetas cada uno) (Ibáñez Martín, 1950, p. 15) 
de científicos varones, aunque también habrá excepciones ${ }^{76}$; caso, por ejemplo, de la valenciana Teresa Bataller quien, tras cursar sus estudios en la Facultad de Medicina de la Universidad de Valencia (obteniendo el Premio Peregrín Casanova) consiguió por oposición, en 1942, la plaza de Médico Femenino del Protectorado para incorporarse después, como Jefe de Laboratorio de la Sección de Neurología, en el Instituto Cajal del CSIC. Allí obtuvo el premio ROEL, en 1948, por su trabajo Influencia de la hipercapnia sobre el cuadro hemático y, dos años antes, su trabajo Estudio experimental del electroshock sobre los centros hemorreguladores y la función suprarrenal fue galardonado con el Premio «Juan de la Cierva». Todas estas credenciales que, junto a la pertenencia al Instituto Español de Fisiología y Bioquímica, nutrían su capital cultural y atribuían de autoridad a la firmante, aparecían, también, convenientemente redactadas en la solapa de la cubierta de ese Premio «Juan de la Cierva», cuya composición, tirada y encuadernación en rústica corrieron a cargo de la Imprenta madrileña de Silverio Aguirre (c/Álvarez de Castro, 38). Un total de 1.000 ejemplares en formato $17 \times 24 \mathrm{~cm}$ que se publicarían a finales de 1950 y con los que el Consejo calculaba sacar «un beneficio sobre el precio de coste de $143,52 \%$ y sobre el precio de venta de 58,9365\%»77.

Por su parte, la pertenencia a la Sección de Espectroscopia del Instituto de Física «Alonso de Santa Cruz», su estancia en la I. G. Fabenindustrie A. G. (Alemania) trabajando bajo la dirección de G. Siebel y K. E. Mann y galardones como el de la Real Academia de Ciencias Exactas, Físicas y Naturales en 1947 serían las credenciales impresas que validarían la autoridad de Antonio Camuñas Puig, también Premio «Juan de la Cierva» (1946) con su trabajo Nuevo Generador para el análisis espectroquímico. Una obra de 56 páginas, en formato $17 \times 24 \mathrm{~cm}$, editada en rústica y con una tirada de 900 ejemplares - con un precio de venta de 12 pesetas - encargada a la Imprenta Talleres Gráficos La Montaña (sita en la calle Amor Hermoso 35, en el madrileño barrio de Usera), que vería la luz, finalmente, en $1951^{78}$.

Este tipo de solapas se incluirían en la mayoría de los Premios publicados por el Consejo: un peritexto editorial ${ }^{79}$ que funcionaba como carta de presentación de los autores, ya fueran estos - o hubieran sido- beca-

\footnotetext{
76 Santesmases, 2000.

77 Archivo Editorial CSIC. Caja 11, Expediente 720

78 Archivo Editorial CSIC. Caja 12, Expediente 755

79 Genette, 2001, pp. 19-35.
} 
rios, ayudantes y finalmente Jefes de Subsección como Alberto Solís García, Premio «Juan de la Cierva» (1947) con Un nuevo sistema de análisis colorimétrico $^{80}$, o perteneciesen al Cuerpo Facultativo de Meteorólogos del Ejército del Aire, doctorándose en Ciencias Físicas por la Universidad de Madrid en 1949 como el guipuzcoano Miguel Azpiroz Yoldi, Premio «Leonardo Torres Quevedo» (1949) con La afinidad en procesos termodinámicos de interés meteorológico -encargado, en el verano de 1950, a los Talleres Gráficos CIES (c/Hermosilla, 135, Madrid) y publicado por el Consejo dos años después ${ }^{81}$.

Otros, en cambio, no necesitaban presentación: no tanto por haber sido pensionado por la JAE, en 1918, para ampliar su formación en el Boureau of Standards de Washington DC y luego en la Universidad de Columbia, o porque fuera catedrático de química en las Universidades de Sevilla y Madrid y director del Instituto de Electrotecnia del CSIC, sino porque, también, era Ministro de Obras Públicas. Hablamos del General José María Fernández-Ladreda (1885-1954), por quien intercedía, el 24 de enero de 1949, su secretario particular, D. Celso Fernández, señalando que «de acuerdo con nuestra conversación telefónica - escribía a Rafael de Balbín - tengo el gusto de decirle que con esta fecha se hace entrega de los CIEN ejemplares al Almacenero de ese Organismo (...) En el dorso de los libros van fijados los precios de venta al público (...) Las liquidaciones está conforme Don José M. en que se hagan en la forma que indica el Sr. Albareda y que sea de la costumbre de Vds.». Liquidaciones por ventas de los 100 ejemplares de cada uno de los volúmenes I, II, III, IV y V de la obra Polvoras y explosivos modernos que se efectuarían «trimestralmente, de acuerdo con nuestras normas, con los descuentos del $35 \%$ concedido a librerías y el $5 \%$ de gastos generales» ${ }^{82}$.

A diferencia de otros autores, Fernández Ladreda sí podía saltarse la cadena de mando, valiéndose para ello de su capital social, y dirigirse directamente al Secretario General del Consejo, el Sr. Albareda, para negociar las condiciones de producción y distribución de unos ejemplares que, por aquel entonces, solicitaron librerías como Livro Ibero Americano (Río de Janeiro) (10 ejemplares el 12/05/1949; 15 ejemplares el 01/07/1949) Teófilo Fernández (Barcelona) (1 ejemplar el 27/06/1949) Librería Casa

\footnotetext{
80 Archivo Editorial CSIC. Caja 15, Expediente 817

81 Archivo Editorial CSIC. Caja 19, Expediente 909

82 Carta entre Celso Fernández y Rafael de Balbín (24 de enero y 12 de marzo de 1949) (Archivo Editorial CSIC. Caja 14, Expediente 795)
} 
Palomo (Almadén) (1 ejemplar el 18/10/1949) Urivelarrea (Buenos Aires) (25 ejemplares el 24/01/1950) o José Pagés (Canarias) (1 ejemplar el 14/02/1950) ${ }^{83}$. Ejemplares de los que, sin embargo, desde el 20 de diciembre de 1950 no se había vendido nada - según apuntaban, en julio de 1951 , desde la Oficina de Publicaciones ${ }^{84}$ - y de los que se vendieron tan sólo 6 ejemplares desde entonces hasta el 31 de diciembre de 1952.

\subsection{Traducciones: una rara avis}

Aquella era, en realidad, una obra originalmente publicada en 1915 y que, junto a Fernández Ladreda, firmaban Jesualdo Martínez Vivas y José Rojas Feigenspán ${ }^{85}$. Lo cierto es que, entre las publicaciones del CSIC, las reediciones fueron siempre más numerosas que las traducciones, que eran entonces, sin embargo, la seña de identidad de las editoriales científicas comerciales españolas ${ }^{86}$. No obstante, cuando se realizaron traducciones, el Consejo las reservó, mayoritariamente, para textos en lengua inglesa o alemana en detrimento de otras lenguas como, por ejemplo, la italiana: «la facilidad de lectura - señalaba José María Otero Navascués (1907-1983) - hace que pueda consultarse directamente y casi sin dificultad, la obra original, como en casos análogos lo demuestra la constante experiencia de este Centro». Argumentos que esgrimía al Secretario General del CSIC, en noviembre de 1941, para desestimar la publicación de la traducción de la obra del profesor Enrico Fermi (1901-1954), Molecole e cristali, realizada por J. Martínez Losada ${ }^{87}$.

Sin embargo, las traducciones en el CSIC siempre tenían su razón de $\operatorname{ser}^{88}$. Véase, por ejemplo, la tirada de 3.000 ejemplares encuadernados en tela de la edición inglesa de ¿Neuronismo o reticulismo? de Santiago Ramón y Cajal (1852-1934) ${ }^{89}$, la cual «se corresponde y se ajusta por completo - decían desde la Oficina de Publicaciones - a la edición española,

${ }^{83}$ Detalles de salidas (18/01/1951) (Archivo Editorial CSIC. Caja 14, Expediente 795)

${ }^{84}$ Nota manuscrita (07/07/1951) (Archivo Editorial CSIC. Caja 14, Expediente 795)

85 Archivo Editorial CSIC. Caja 14, Expediente 795

86 García Naharro, 2019.

${ }^{87}$ Carta de José M. ${ }^{\text {a }}$ Otero al Secretario General del CSIC (21 de noviembre de 1941) (Archivo Biblioteca Rocasolano. Carpeta Libros 1941)

88 Para una aproximación analítica al fenómeno de las traducciones y la circulación internacional del conocimiento véase Heilbron; Sapiro 2007.

89 Mainer, 2006. 
aprovechando los grabados de ésta $»^{90}$. El prestigio internacional de Cajal era un activo que, de cara al extranjero, la dictadura nunca perdió de vista, traduciendo su obra y relacionando su imagen y su nombre a los del Consejo, el organismo editor de estos ejemplares puestos a la venta al precio de 58 pesetas, y que constaban de 170 páginas y 71 figuras intercaladas en el texto. Publicados en 1954, la traducción corrió a cargo del Reverendo Padre Manuel Úbeda (1913-1999), Director del Colegio Mayor Santo Tomás de Aquino, cuya gratificación - como traductor y editor de la edición inglesa Neuron Theory or Reticular Theory? - seguía sin cobrar en enero de 1957, cuando Rafael de Balbín, Jefe de la Sección de Publicaciones del Consejo, le aseguraba que «ya está enmendado este olvido, y pienso que de aquí a unos días, la Comisión Permanente aprobará la gratificación propuesta de 8.440 pesetas y 55 céntimos. Hemos aplicado la tarifa que por aquí tenemos, pero te ruego que si consideras insuficiente esta gratificación, me lo indiques en la forma que te sea más cómoda». Una edición con vistas a la exportación cuya salida, sin embargo, a finales de los años cincuenta, no había sido «excesiva», "puesto que de los 3.000 ejemplares que se hicieron - escribía Rafael de Balbín - quedan más de 2.000 en el almacén todavía» ${ }^{91}$.

Otras traducciones tenían, en cambio, una finalidad diferente: caso de Fundamentos matemáticos de la mecánica cuántica, original (publicado en Berlín en 1932 por J. Springer) de John von Neumann (1903-1957) y traducido del alemán por el Dr. Ramón Ortiz Fornaguera (1916-1974) ${ }^{92}$, que cobraría 3 céntimos por cada una de las 94.000 palabras del original alemán ${ }^{93}$ y de quien Esteban Terradas (1883-1950) decía que había realizado su tarea «con ejemplar fidelidad, reverente atención y conocimiento adecuado del tema». Terradas, científico eminente que había sido «expulsado» ${ }^{94}$ ya por dos veces de la universidad española, tras regresar a ella hacía esta reflexión en el prólogo a la versión española de esta obra, «costeada por el Consejo de Investigaciones Científicas; (que por su natu-

\footnotetext{
90 Informe de 10 de julio de 1952 (Archivo Editorial CSIC. Caja 41, Expediente 1700)

91 Carta de Rafael de Balbín al P. Manuel Úbeda (24/01/1957) (Archivo Editorial CSIC. Caja 41, Expediente 1700).

92 Soler Ferrán, 2015, pp. 9-55.

93 En total cobraría en dos recibos, uno de 2.820 pesetas y otro de 6.000 pesetas, por la traducción del cuerpo y las anotaciones de la obra original de Johann von Neuman (3 de marzo de 1948). (Archivo Editorial CSIC. Caja 12, Expediente 742).

94 Roca Rosell, 1990, pp. 71-104; Rey Pastor, 1951, pp. 35-64.
} 
raleza) no hubiera sido posible confiarla a un editor ávido de ganancia» y cuya finalidad, decía, era ser «comentada durante cursos sucesivos en el Seminario de Física Matemática de la Universidad de Madrid». Una versión que, además, tal y como recordaba:

Comenzó muy antes de aparecer la edición francesa, con el objeto de que nuestros estudiantes dispusieran de una relación matemática de la Teoría de los Quanta como fundamento lo más riguroso posible de las aplicaciones de la misma a los diversos problemas de la Física atómica y como necesaria educación del pensamiento para aplicar concienzudamente los principios generales en que se apoy $\mathrm{a}^{95}$.

Una obra de carácter teórico y profundamente matemático, que clarificaba las últimas aportaciones teóricas a la mecánica cuántica y que Terradas pretendía emplear con sus alumnos de la Universidad de Madrid; un espacio selecto ${ }^{96}$ en el que, en la práctica y según parece, no hubo siempre plena concordancia con los postulados ideológico-culturales del nacionalcatolicismo ${ }^{97}$. Las inquietudes personales de Terradas y de Ortiz Fornaguera motivaron la publicación de esta traducción, realizada por el que fuera profesor de la Universidad de Barcelona, de la Escuela Técnica Superior de Ingenieros de Armas Navales y trabajador del Instituto de Óptica «Daza de Valdés» (entre otras instituciones), y que fue, también, antiguo alumno de Terradas (quien llegó a decir de él: «en Física Matemática es el mejor alumno que he tenido» ${ }^{98}$ ). En la mencionada traducción, Ortiz Fornaguera también dejó su impronta como ese otro autor que es siempre el traductor ${ }^{99}$ : no sólo mediante las pertinentes notas al pie, sino también en la definición de ciertos operadores funcionales que, como en otros casos particulares, aparecieron publicados modificando la redacción original de von Neumann ${ }^{100}$.

95 Prólogo de la traducción española de Neumann, 1949.

96 Sobre la circulación de la información científica en la España franquista y el contraste radical entre la esfera pública y los círculos elitistas véase Florensa, 2017.

${ }^{97}$ Otero Carvajal, 2014, pp. 131-190.

98 Baig i Aleu; Valentín-Gamazo; Gómez del Moral, 2012, p. 182.

99 Sobre la figura de los traductores de libros técnicos remitimos al caso de los traductores de la editorial Gustavo Gili en García Naharro, 2019, pp. 75-133.

100 Algunas de sus modificaciones aparecerán publicadas en la versión inglesa de 1955 traducida por Robert T. Beyer y editada en Princeton (Baig i Aleu; Valentín-Gamazo; Gómez del Moral, 2012, pp. 188-189). 
El coste de la composición, ajuste y tirada (sin encuadernación ni papel) para los 2.000 ejemplares previstos lo fijó la Imprenta Nuevas Gráficas S.A. en 1.100 pesetas por cada pliego de 16 páginas, «por tratarse de una obra matemática enormemente difícil». «En el precio está incluido - apuntaban desde la Imprenta - el valor de las matrices que serán necesarias hacer de signos matemáticos no corrientes en España» ${ }^{101}$. En vista de ello, la tirada se redujo a 1.500 ejemplares para los que se encargaron 30 remesas de papel que, no obstante, fueron finalmente insuficientes. Así, el 7 de julio de 1949, la Imprenta solicitaba seis remesas y media más al Consejo que, por aquel entonces, no tenía «en almacén papel que se asemeje al servido para la tirada», rogándoles, por tanto, que fueran ellos quienes se encargaran de «encontrar esas seis y media remesas que son necesarias para su terminación, procurando que sean lo más exactamente análogas a las ya enviadas» ${ }^{102}$.

Esa solía ser la política seguida, entonces, desde el Servicio de Publicaciones del Consejo: encargar el presupuesto por composición, ajuste y tirada (sin papel, que se encargaba aparte) más el de encuadernación —en rustica o tela - incluyendo, generalmente, el valor de las cubiertas:

Te envío la carpeta de presupuestos de encuadernación, según se lo has indicado a Flores - escribía Rafael de Balbín Lucas a Tomás Alvira, secretario del Instituto Español de Edafología y Fisiología VegetalRespecto de ellos, hay, como verás, una notable diferencia en los precios, que alcanza a dos pesetas por ejemplar. El más barato es el de Fernández, y además su presentación es sin duda superior a la de Duque. En cuanto al presupuesto de Montaña, es el más caro; no ha encuadernado nunca para el Consejo, y creo que una prudencia elemental aconseja en todo caso pedirle la muestra de la encuadernación. Estas son las normas corrientes en Publicaciones, y te las comunico para tu orientación ${ }^{103}$.

Normas que seguiría el secretario del citado Instituto para la edición del libro Claves sistemáticas del suelo, del profesor Walter Kubiëna (1897-1970) y que, «por haberse realizado tres ediciones simultaneas en

101 Presupuesto de 24 de febrero de 1948 (Archivo Editorial CSIC. Caja 12, Expediente 742).

102 Cartas de 7 de julio y 9 de Julio de 1949 (Archivo Editorial CSIC. Caja 12, Expediente 742).

103 Carta de Rafael de Balbín a Tomás Alvira (20 de diciembre de 1952) (Archivo Editorial CSIC. Caja 21, Expediente 933). 
los idiomas español, inglés y alemán y por ser un libro de interés exclusivo de los que cultivan la Ciencia del Suelo, hacen que creamos oportuno - escribía el director del Instituto y Secretario General del CSIC, José M. ${ }^{a}$ Albareda - realizar una experiencia de propaganda y venta de esta obra por el Instituto de Edafología ${ }^{104}$. Una obra costeada, sin embargo, por la Oficina de Publicaciones, quedando así el Instituto «en situación deudora con Publicaciones por el importe de la impresión» ${ }^{105}$.

A finales del pasado mes de enero fue terminada de imprimir la obra CLAVES DE LOS SUELOS, del Prof. Kubiena (sic) e inmediatamente, según órdenes recibidas, fueron encuadernados 500 ejemplares de esta obra, en rústica, ejemplares que - escribían desde Talleres Gráficos Montaña - todavía están en poder de nuestro encuadernador (...) Asimismo tenemos el resto de la obra sin entregar por faltar también que nos indiquen dónde la entregamos (...) nos permitimos molestar su atención sobre esto, ya que la obra se perjudica con este prolongado apilamiento e incluso puede llegar a estropearse ${ }^{106}$.

El montante total de la edición española sumaba 3.000 ejemplares, 388 páginas con 12 figuras en el texto para las que hicieron falta 76 remesas de papel. Números similares en los que se movieron las ediciones inglesa y alemana, en su caso impresas por la casa madrileña Gráficas Reunidas S.A. (fusión de las antiguas casa Raoul Peant, Julián Palacios y Bernardo Rodríguez) (c/Hermosilla, 110): 3.500 ejemplares de 392 páginas, 26 láminas y 12 figuras en el texto para la edición alemana y 3.500 ejemplares de 320 páginas, 26 láminas y 12 figuras en el texto para la edición inglesa - traducida por Tomás Alvira-, de los cuales, 300 había que «situar lo antes posible en Encuadernación Fernández» (Taller de Encuadernación Francisco Fernández, c/Larra, 11, Madrid), pues la distribuidora inglesa George Allen \& Unwin Ltd. empezaba a impacientarse ${ }^{107}$. Sin embargo, todavía habría rectificaciones que acometer.

104 Carta de Albareda al Jefe de la Sección de Publicaciones (17 de marzo de 1953) (Archivo Editorial CSIC. Caja 21, Expediente 933).

105 Carta de Albareda a Rafael de Balbín (18 de marzo de 1953) (Archivo Editorial CSIC. Caja 21, Expediente 933).

106 Carta de Talleres Gráficos Montaña al Jefe de Publicaciones del CSIC (7 de abril de 1953) (Archivo Editorial CSIC. Caja 21, Expediente 933).

107 Carta de George Allen \& Unwin Ltd a la Secretaria del CSIC (23 de marzo de 1953) Nota de Sección Ediciones (Almacén) (20/05/1953) (Archivo Editorial CSIC. Caja 21, Expediente 933). 
Tal y como aparece editado este libro, en sus distintas versiones española, inglesa y alemana, requiere modificaciones imprescindibles si se desea dejar a salvo el prestigio del C.S.I.C. como editor, así como dar a las tres ediciones una unidad y una base mínima de garantía editorial y científica ${ }^{108}$.

Así comenzaba el informe sobre la edición de la obra del profesor $\mathrm{Ku}$ biëna, donde se apuntaban una serie de modificaciones indispensables; entre otras, el sustituir, en la sobrecubierta de papel y en la cubierta, el nombre del distribuidor inglés y alemán por el nombre del Consejo ${ }^{109}$, cuyas siglas acompañarían, también, al apellido del autor y al título en inglés - o en alemán - del libro en el lomo. También, como venía siendo habitual, se volvía a demandar el situar el exlibris del Consejo en la última página de la obra y en la contraportada (no en negro, como figuraba, sino en oro). Ni sobrecubierta ni portada consideraban que fueran «lugares adecuados para hacer figurar el año de la edición», al tiempo que se apuntaba la necesidad de «unificar el material de encuadernación» y la tipografía de las encuadernaciones en tela siguiendo el modelo de la edición alemana. A su vez, el nombre del traductor - entendido «como garantía científica de la versión inglesa»- debía aparecer en la portadilla dejando «cinco centímetros de espacio encima del pie editorial, con objeto de que el distribuidor inglés pueda, si así lo desea, estampar en los ejemplares que vaya recibiendo un sello de caucho haciendo constar su nombre y su calidad de distribuidor exclusivo de la edición inglesa», como también se haría en la edición alemana ${ }^{110}$.

Todos ellos, de nuevo, nombres propios, de empresas e instituciones situados en diferentes espacios y cumpliendo funciones específicas. Elementos textuales y paratextuales al servicio de toda una serie de actores - principales o secundarios - que ocupan su lugar en la geografía espacial del objeto impreso y cumplen su papel en la compleja economía de nombres que siempre está detrás de la construcción de la función autor de una obra científica ${ }^{111}$ como ésta, por la que, ya en julio de 1954, se intere-

108 Informe sobre la obra «Claves sistemáticas del suelo» del Profesor W. L. Kubiena (Archivo Editorial CSIC. Caja 21, Expediente 933).

109 «Se propone poner con todas sus letras el nombre del Consejo, ya que las iniciales «C.S.I.C» no pueden decirle gran cosa al lector inglés» (Informe, p. 1).

110 Informe sobre la obra «Claves sistemáticas del suelo» del Profesor W. L. Kubiena (Archivo Editorial CSIC. Caja 21, Expediente 933).

111 Biagioli, 2006, p. 134-137. 
saba el editor holandés J. P. Coelingh, director de la Casa Meulenhoff y Compañía, para una posible traducción al holandés ${ }^{112}$.

\section{Conclusiones: reconstruyendo a tientas las prácticas de la edición científica en la España franquista}

Así, tal y como hemos comprobado, los manuscritos científicos no están sólo condicionados por el orden del discurso ${ }^{113}$, sino también por las vicisitudes propias de su proceso de conversión de meros escritos a libros completos. Obras impresas que, durante su confección, pasarán por diversas manos, las de los diferentes actores inmersos en la cadena de publicación que, desde nuestra perspectiva de análisis, más que en cadena de suministro se ha tornado en un encadenamiento de acciones que aportan valor al producto final ${ }^{114}$, fruto de la interacción productiva de operarios, máquinas, empresas e instituciones que forman parte del proceso, siempre colectivo, de la edición del libro.

Un proceso por el cual los textos pasan a dotarse de un formato que, unido al estatus social del mismo y de los nombres a los que se liga, condiciona la forma en que éstos son recibidos $-\mathrm{y}$, en el mejor de los casos, leídos. Toda una coreografía de nombres distribuidos espacial, tipográfica y textualmente en torno a la propia composición del soporte impreso. Junto a todo ello y como elementos transversales, cuestiones de fondo en torno a la figura del científico como autor o a la construcción de la autoridad científica en la España franquista han estado presentes, también, a la hora de acercarnos a esos textos ensamblados en libros que, como hemos visto, adquieren valor, reconocimiento y credibilidad a través de su relación material con los diferentes nombres propios y de instituciones a los que se engarzan.

En definitiva, a lo largo de estas páginas hemos tratado de ver cómo funcionan esos nombres ensamblados en los objetos impresos puestos en circulación y articulados sobre relaciones sociales desplegadas en el contexto dictatorial y espacio-temporal de la España franquista. Partiendo de esas premisas, nos hemos acercado a algunas de las monografías publica-

112 Carta del Asesor Técnico de Publicaciones (José Miguel de Azaola) a Tomás Alvira (16/VII/1954) (Archivo Editorial CSIC. Caja 21, Expediente 933).

113 Foucault, 1992.

114 Thompson, 2012, pp. 3-22. 
das por el CSIC, la institución nacida «bajo el signo del Caudillo» ${ }^{115}$ y cuya labor editorial nació «también marcada con el signo unitario y profundo de algunos grandes empeños culturales» ${ }^{116}$. Así se refería el presidente del CSIC, José Ibáñez Martín, a la empresa corporativa y nacional de la edición científica del Consejo, cuyas publicaciones, desde abril de 1944, quedaron exentas del trámite generalizado de censura previa, si bien es cierto que éstas venían siendo ya autorizadas de facto, «sin pasar por el lectorado ni atravesar la línea del despacho ordinario», desde tiempo atrás ${ }^{117}$.

Publicaciones con las que, como hemos visto, se daba voz a las autoridades de la ciencia en España: personalidades competentes científicamente y reconocidas políticamente que coparán las páginas de las revistas y monografías del CSIC $^{118}$, el organismo oficial que rubricará y dotarán de capital simbólico a sus nombres en una España dictatorial donde el Estado era la instancia última de validación. Al mismo tiempo, tanto el CSIC como el resto de instituciones científicas oficiales eran también las encargadas en aquella España de dar el visto bueno - mediante un informe del responsable del Centro correspondiente (Institutos y Centros del CSIC, Universidades del Estado o Escuelas especiales superiores) - a las obras publicadas por las empresas editoriales privadas, convirtiéndose así, a la postre, en los valedores últimos de los trabajos científicos y técnicos que aparecerían publicados en el ámbito comercial español. Todo ello hará, también, que tanto los autores como los productos impresos de la ciencia oficial gocen de un status privilegiado ante un mundo editorial privado que buscará contar siempre con su beneplácito ${ }^{119}$.

No obstante, eso no significa que ni las personalidades del CSIC ni sus publicaciones estuvieran exentas de enfrentarse a los contratiempos propios del mundo editorial: un universo pródigo en situaciones paradójicas aunque irremediablemente cotidianas en el quehacer diario del oficio ${ }^{120}$. De ahí que, a pesar de contar con los recursos y las facilidades que la dictadura les ofrecía, las publicaciones del CSIC estuvieran también a expensas de los tiem-

115 CSIC, 1942, p. V.

116 Discurso de José Ibáñez Martín en la Solemne Sesión de Clausura del XI Pleno del Consejo Superior de Investigaciones Científicas bajo la presidencia del Jefe del Estado (3 de febrero de 1951) (CSIC, 1951b, p. 69.)

117 Ruiz Bautista, 2005, p. 277.

118 Malet, 2008; Canales, 2019.

119 García Naharro, 2019a.

120 García Naharro, 2019b. 
pos del proceso de edición y las modificaciones presupuestarias que estos podían conllevar, tal y como ocurriera, por ejemplo, con La interpretación geológica de las mediciones geofísicas de José García Siñeríz (1886-1974), director del Instituto Nacional de Geofísica; una obra que se vio alterada por las restricciones eléctricas que sufrió, en 1949, la imprenta Sucesores de Rivadeneyra S.A. (Paseo de Onésimo Redondo, 26. Madrid) ${ }^{121}$.

Otras veces, en cambio, el retraso en la publicación podía deberse una actuación deficiente del CSIC a la hora de suministrar, en tiempo y forma, el papel correspondiente a la imprenta: caso del segundo tomo de Dinámica Cerebral del Doctor Justo Gonzalo (1910-1986), quien sufrió por ello «un gran trastorno en las clases» porque, decía, había «contado con este nuevo tomo para mi curso actual (1948) del Doctorado en la Facultad» ${ }^{122}$. Cuestiones en apariencia tan banales como el tipo de papel y el precio, o incluso el propio formato serán, tal y como hemos visto, un motivo de disputa: así ocurrió también con el libro del CSIC, Introducción a la Astronomía, cuya primera edición no tenía solapas y, por tanto, tampoco referencia alguna al autor de la obra, el capellán Ramón María Aller (1878-1966) ${ }^{123}$. Aunque, en cualquier caso, los jueces últimos de todo libro fueron (y son) siempre los lectores, esos cuya opinión motivó, por ejemplo, que, en diciembre de 1954, el germen del futuro libro La técnica del análisis factorial de Mariano Yela Granizo (1921-1994) llevara «infinidad de tiempo parado» ${ }^{124}$.

Unos lectores que, en aquellos años, podían encontrar las publicaciones del CSIC, principalmente, tanto en las bibliotecas de los Centros de Investigación como en la Librería Científica Medinaceli (c/ Duque de Medinaceli n. ${ }^{\circ}$ 4, Madrid), el establecimiento oficial del CSIC - el «hogar para los lectores cultos, diríamos sabios»- inaugurado en 1950 por José Ibáñez Martín y que contaba, entonces, tanto «en escaparates, mostradores y plúteos» con «ejemplares de 1.196 obras y 80 revistas de las

121 «Nos vemos precisados a manifestarles que los precios consignados tendrán un recargo del 15\% en concepto de Grupo Electrógeno». Carta 6 de agosto de 1949 (Archivo Editorial CSIC. Caja 2, Expediente 378).

${ }^{122}$ Cartas a Balbín y Flores de 4 de enero de 1948 y 26 de noviembre de 1948 respectivamente (Archivo Editorial CSIC. Caja 5 Expediente 487).

123 García Naharro, 2015a, 774-775.

124 «Según las observaciones que me hicieron los alumnos y profesores de psicología y pedagogía, a los cuales estaba principalmente destinado el libro, los artículos resultaban difíciles de comprender sin una preparación matemática especial». Informe Publicación Análisis Factorial (31 de diciembre de 1954)(Archivo Editorial CSIC. Caja 26, Expediente 995). 
editadas por nosotros» ${ }^{125}$. Todo ello en un tiempo donde, sin embargo, ni todos los lectores ni todas las publicaciones eran iguales. No olvidemos, por ejemplo, que por Orden del 18 de febrero de 1941 -firmada por José Ibáñez Martín - las publicaciones del CSIC fueron de obligada adquisición «con carácter preferente a las demás» para todos los Centros de Enseñanza Media y Superior, así como para las «Bibliotecas Universitarias, Provinciales y Populares» ${ }^{126}$. Así mismo, a la publicidad gratuita y constante de las cadenas de radiodifusión y prensa del Movimiento con la que contaban catálogos como el de Editora Nacional, se unía la obligación de mantener visibles las publicaciones de la Vicesecretaría de Educación Popular en el interior de todas las librerías de España, contando además, en todas ellas, con una semana de propaganda en donde al menos uno de sus escaparates (o una parte importante de éste, si sólo disponían de uno) debía reservarse para la exposición de sus publicaciones ${ }^{127}$.

Aunque, sin embargo, no siempre lo esencial será lo más visible. Así, mientras en los años sesenta y en el escaparate de la librería de Siegfried Blume - distribuidor y dueño de la barcelonesa Librería Técnica Extranjera - figuraban obras «de las principales editoriales nacionales, europeas y americanas», no es menos cierto que, en su trastienda, podían encontrarse, también, libros de editoriales disidentes como la antifranquista Ruedo Ibérico. Por ello, no debemos olvidar que productos impresos, editoriales y librerías fueron también cauces de incursión, en España, de toda una línea de publicaciones de oposición a la dictadura ${ }^{128}$. Pero eso, claro está, es ya otra historia.

\section{Fuentes y archivos}

Archivo General de la Administración (AGA)

Archivo General de la Universidad de Navarra (AGUN)

Archivo de la Editorial del Consejo Superior de Investigaciones Científicas (CSIC)

Archivo de la Biblioteca Nacional de España (BNE)

125 Boletín Bibliográfico del Consejo Superior de Investigaciones Científicas, N. ${ }^{\circ} 55$, 1950 , p. 4 ; p. 9.

126 Orden de 18 de febrero de 1941 (BOE 28/02/1941)

127 Ruiz Bautista, 2005, p. 219.

128 García Naharro, 2015a, p. 775. Cornellà-Detrell, 2021. Sobre publicaciones clandestinas véase también Martínez Martín, 2016. 


\section{Bibliografía}

Albareda, José María, Panorama de la investigación en España, Madrid, CSIC, 1964.

Baig I Aleu, Marià; Valentín-Gamazo, Gonzálo G.; Gómez del Moral, Mercè X: «Von Neumann traducido por Ortiz: una obra pionera en la enseñanza de la cuántica» en HERRAN, Nestor y RoQué, Xavier (eds.), La física en la dictadura. Físicos, cultura y poder en España 1939-1975, Bellaterra, Universitat Autónoma de Barcelona, 2012, pp. 182.

Barona, Josep Lluis, El exilio científico republicano, Valencia, Publicacions de la Universitat de València, 2010.

Biagioli, Mario; Galison, Peter (eds.), Scientific Authorship. Credit and Intellectual Property in Science, London, Routledge, 2003.

Biagioli, Mario, «Documents of documents. Scientists'Names and Scientific Claims», en RILE Annelise (ed.), Documents: Artifacts of modern knowledge, Michigan, The University of Michigan Press, 2006, pp. 129156.

BouRdieu, Pierre, Las reglas del arte. Génesis y estructura del campo literario, Barcelona, Anagrama, 1995.

Bourdieu, Pierre; Chartier, Roger, «La lectura: una práctica cultural», en Bourdieu Pierre, El sentido social del gusto. Elementos para una sociología de la cultura, Buenos Aires, Siglo XXI, 2010, pp. 253-274.

Bourdieu, Pierre, Intelectuales, política y poder, Barcelona, Anagrama, 2012.

Bouveresse, Jacques, Prodigios y vértigos de la analogía. Sobre el abuso de la literatura en el pensamiento, Buenos Aires, Libros del Zorzal, 2001.

Box, Zira, «Los atributos de la nación. Género y clase en la España franquista» en LEMUs, Encarnación; PEÑA, Manuel (eds.), Alianzas y propaganda durante el primer franquismo, Barcelona, Planeta, 2019, pp. 125-146.

ButLER, Judith, Bodies that matter. On the discursive limits of «sex», Routledge, New York/London, 1993.

CABAllero Garrido, Ernesto (coord.), La Junta para Ampliación de Estudios e Investigaciones Científicas: historia de sus centros y protagonistas (19071939), Madrid, Trea, Asociación Nacional de Estudiantes e Investigadores siglo XXI, 2010.

CAMPrubí, Lino, Engineers and the Making of the Francoist Regime, Massachusetts, MIT Press, 2014.

Claret MirAnda, Jaume, El atroz desmoche. La destrucción de la universidad española por el franquismo, 1936-1945, Barcelona, Crítica, 2006.

CANAlES, Antonio Fco., «The reactionary Utopia: the CSIC and Spanish Imperial Science» en Gómez, Amparo; CANAles Antonio Fco. y Balmer Brian (ed.), Science Policies and Twentieth-Century Dictatorships. Spain, Italy and Argentina, Farnham, Ashgate, 2015, pp. 79-102. 
Ciencia de papel. Privilegios y autoridad en España durante el primer franquismo (1939-1959)

CANales, Antonio Fco., «El CSIC en el sistema de I+D español desde su creación al tardo-franquismo» en Delgado, Lorenzo; López, Santiago M. (eds.), Ciencia en transición. El lastre franquista ante el reto de la modernización, Madrid, Silex, 2019, pp. 39-62.

CORnEllà-Detrell, Jordi., «Lectores, libreros y distribuidores ante la represión cultural: la circulación de libros clandestinos durante el franquismo» en Agustí, Lluís; Baró, Mónica; Rueda Ramírez, Pedro (eds.), Redes del libro en España. Agentes y circulación del impreso (siglos XVII-XX), Zaragoza, Prensas de la Universidad de Zaragoza, 2021, pp. 341-364.

CSIC, Memoria de la Secretaría General (1940-41) Madrid, CSIC, 1942.

CSIC, Memoria de la Secretaría General (1942) Madrid, CSIC, 1943.

CSIC, Memoria de la Secretaría General (1943), Madrid, CSIC, 1944.

CSIC, Memoria de la Secretaría General (1944) Madrid, CSIC, 1945.

CSIC, Memoria de la Secretaría General (1945), Madrid, CSIC, 1946.

CSIC, Memoria de la Secretaría General (1946-1947) Madrid, CSIC, 1948.

CSIC, Memoria de la Secretaría General (1948) Madrid, CSIC, 1950.

CSIC, Memoria de la Secretaría General (1949), Madrid, CSIC, 1951.

CSIC: MEMoria de LA SECRETARÍa General (1950) MAdRID, CSIC, 1951b

CSIC, Memoria de la Secretaría General (1951), Madrid, CSIC, 1952.

Delgado, Lorenzo; López, Santiago M. (eds.), Ciencia en transición. El lastre franquista ante el reto de la modernización, Madrid, Silex, 2019.

DíAZ-PINES Y FERNÁNDEZ PACHECO, Onésimo, «El Consejo Superior de Investigaciones Científicas», Temas Españoles, 82, 1959.

Florensa, Clara, Els discursos sobre l'evolució en el franquisme (1939-1967). La Generación del 48 i la evolución sin problema, Barcelona, UAB, 2017.

Formentin IbÁÑEZ, Justo; CARrascosA, Alfonso V.; RodrigueZ Fraile, Esther, José Ibáñez Martín y la ciencia española: el Consejo Superior de Investigaciones Científicas, Madrid, CEU, 2015.

Foucault, Michel, El orden del discurso, Buenos Aires, Tusquets, 1992.

Frasca-EsPadA, Marina; JARDINE, Nick (eds.), Books and the Sciences in History, Cambridge, Cambridge University Press, 2000.

GARCía NAHARRO, Fernando, «La edición académica. Las ediciones científicas y el libro técnico», en Martínez Martín, Jesús A. (coord.), Historia de la edición en España (1939-1975), Madrid, Marcial Pons, 2015a, pp. 759-782.

GARCía NAHARRO, Fernando, «Las publicaciones oficiales. Editora nacional», en Martínez Martín, Jesús A. (coord.), Historia de la edición en España (1939-1975), Madrid, Marcial Pons, 2015b, pp. 209-232.

García Naharro, Fernando, El papel de la ciencia. Publicaciones científicas y técnicas durante el franquismo (1939-1966), Madrid, UCM, 2017.

GARcía NAHARro, Fernando, Editando ciencia y técnica durante el franquismo. Una historia cultural de la editorial Gustavo Gili (1939-1966), Zaragoza, Prensas de la Universidad de Zaragoza, 2019a. 
García NAharro, Fernando, «Irremediablemente cotidiano. Prácticas comerciales en Editora Nacional», Bulletin d'histoire contemporaine de l'Espagne, 53, 2019b, pp. 257-271.

GARCía NAHARRo, Fernando, «Lectores extranjeros de obras científicas y técnicas durante el Franquismo: Cartas al Servicio Nacional de Información Bibliográfica (1952-1966)», Pasado y memoria, n. ${ }^{\circ}$ 21, 2020a, pp. 105-130.

García NAHARro, Fernando, «El "audible silencio" durante el Franquismo: artículos científicos y técnicos publicados en revistas y lenguas extranjeras por investigadores del CSIC (1939-1964)», Llull, Vol. 43 (N. ${ }^{\circ} 87$ ) 2020b.

García Naharro, Fernando, «In the Land of Men: Women in Applied Sciences at the CSIC», en Janué i Miret, Marició; Presas i Puig, Albert (eds.), Science, Culture and National Identity in Francoist Spain, 1939-1959. Cham, Palgrave Macmillan, 2021a, pp. 155-175.

GARCía NAHARro, Fernando, «Reshaping Scientific Journals. Applied Science and its audience under Franco (1939-1966)», Journal of Iberian and Latin American Studies, 27:1, 2021b, pp. 115-132

GARCÍA NAHARRo, Fernando, Palabras, actores, objetos y lugares. El CSIC y la demarcación de la ciencia en la España franquista (1939-1966), Madrid, CSIC, 2022 (en prensa).

Genette, Gérard, Umbrales, México, Siglo XXI, 2001, pp. 227-246.

Gili, Mónica; Puente, Moisés; Rojals, Marta (eds.), Editorial Gustavo Gili. Una historia 1902-2012, Barcelona, Gustavo Gili, 2013.

GonZÁlez Blasco, Pedro, El investigador científico en España, Madrid, CIS, 1980.

González Blasco, Pedro; Jiménez Blanco, José, «La investigación en el Consejo Superior de Investigaciones Científicas. Estudio de un grupo significativo durante el periodo 1940-1955», en González Blanco, Pedro; JiméNEZ Blanco, José; LóPez Piñero, José María, Historia y sociología de la ciencia en España, Madrid, Alianza, 1979, pp. 126-163.

Gordin, Michael; Grunden, Walter; WALKER, Mark; WANG, Zuoyue, «Ideologically Correct» Science», en WALKER, Mark (ed.), Science and ideology. A Comparative History, London, Routledge, 2003, pp. 35-65.

Guston, David H, Between politics and science, Cambridge, Cambridge University Press, 2006.

GutiÉRrez Ríos, Enrique, José María Albareda: una época de la cultura española, Madrid, CSIC, 1970.

Haraway, Donna J., «Modest Witness: Feminist Diffractions in Science Studies», en Galison, Peter; Stump, David J. (eds.), The Disunity of Science. Boundaries, Contexts and Power, Stanford, Stanford University Press, 1996, pp. 428-442.

HeIlbron, Johan; SAPIRO, Gisèle, «Outline for a sociology of translation. Current issues and future prospects», en WOLF, Michaela; FUKARI, Alexandra 
(eds.), Constructing a Sociology of Translation, Amsterdam/Philadelphia, John Benjamins Publishing Company, 2007, pp. 93-108.

Herran, Nestor; Roqué, Xavier, «Los físicos en el primer franquismo: conocimiento, poder y memoria», en HERRAN, Nestor y RoQuÉ, Xavier (eds.), La física en la dictadura. Físicos, cultura y poder en España 1939-1975, Be1laterra, Universitat Autónoma de Barcelona, 2012, pp. 85-104.

Herran, Nestor; RoQué, Xavier, «An Autarkic Science: Physics, Culture, and Power in Franco's Spain», Historical Studies in the Natural Sciences, Vol. 43, No. 2 (April 2013), p. 209.

IbÁÑEZ Martín, José, X Años de Servicios a la Cultura Española 1939-1949, Madrid, Hijos de Heraclio Fournier/Editorial Magisterio Español, 1950.

Instituto NACIONAL DE EstadísticA, Estadística de la producción y comercio del libro español. Años 1946-1957, Madrid, INE, 1958.

JASANOFF, Sheila (ed.), States of knowledge. The co-production of science and social order, London, Tylor \& Francis, 2004.

JoHns, Adrian, The Nature of the Book. Print and Knowledge in the making, Chicago, University of Chicago Press, 1998.

LAtour, Bruno, Aramis or the love of technology, Cambridge, Harvard University Press, 1996.

LóPEz SÁNCHEZ, José María, Los refugios de la derrota. El exilio científico e intelectual republicano de 1939, Madrid, La Catarata-CSIC, 2013.

López SÁnchez, José María; Férnandez Gallego, Alba, A imprenta y tírese. 80 años de la Editorial CSIC, Madrid, CSIC, 2021.

MAINER, José Carlos (ed.), Cajal: una reflexión sobre el papel social de la ciencia, Zaragoza, CSIC, 2006.

MALET, Antoni, «Las primeras décadas del CSIC: investigación y ciencia para el franquismo», en Romero de PABlos, Ana; SANTESMASES, María Jesús (eds.), Cien años de política científica en España, Bilbao, Fundación BBVA, 2008, pp. 211-256.

Malet, Antoni, «José María Albareda (1902-1966) and the Formation of the Spanish Consejo Superior de Investigaciones Científicas», Annals of Science, 66, 2009, pp. 307-332.

Marset Campos, Pedro; Valera Candel, Manuel; Palao Poveda, Gerardo; LÓPEZ FERNÁNDEZ, Carlos, «Aproximación a la investigación química española desde 1940 a 1965 a través de los Anales de la Real Sociedad Española de Física y Química», Llull, vol. 7, 1984,pp. 25-46.

Martínez Martín, Jesús A. (com.), Letras clandestinas 1939-1976. Catálogo Exposición, Madrid, Imprenta Municipal, 2016.

MCKenZIE, D. F., Bibliografía y sociología de los textos, Madrid, Akal, 2005.

Ministerio DE INFORMACIÓN Y TURISMO, Libros técnicos y científicos, INLE, Madrid, 1966. 
Von Neumann, John, Fundamentos matemáticos de la mecánica cuántica, Madrid, Instituto Jorge Juan, 1949.

NIETO, Alejandro: «Presentación» en NIETO, Alejandro et al. (coords.): Apuntes para una política científica. Dos años de investigación en el CSIC: 1980-1982, Madrid, CSIC, 1982, pp. 11-14.

Nieto-Galan, Agustí, The Politics of Chemistry. Science and Power in Twentieth-Century Spain, Cambridge, Cambridge University Press, 2019.

Otero Carvajal, Luis Enrique; LóPez SÁnchez, José María, La lucha por la modernidad. Las ciencias naturales y la Junta para Ampliación de Estudios, Madrid, CSIC-Amigos de la Residencia de Estudiantes, 2012.

Otero Carvajal, Luis Enrique, La destrucción de la ciencia en España, Madrid, Editorial Complutense, 2006.

Otero CARVAJAL, Luis Enrique (dir.), La Universidad nacionalcatólica. La reacción antimoderna, Madrid, Universidad Carlos III, 2014.

Otero Carvajal, Luis Enrique, «La Física y las Matemáticas en la universidad nacional católica», en OTERO CARVAJAL, Luis Enrique (dir.), La Universidad Nacionalcatólica, la reacción antimoderna, Madrid, Universidad Carlos III de Madrid, 2014, pp. 131-190.

Pimentel, Juan, Fantasmas de la ciencia española, Madrid, Marcial Pons, 2020.

PRESAS I PUIG, Albert, «Nota histórica: una conferencia de José María Albareda ante las autoridades académicas alemanas», Arbor, CLX, 631-632 (JulioAgosto 1998), pp.

PResas I Puig, Albert, «La correspondencia entre José M. Otero Navascués y Karl Wirtz, un episodio de las relaciones internacionales de la Junta de Energía Nuclear», Arbor, CLXVII, 659-660 (Noviembre-Diciembre 2000), pp. 527-601.

Puig-SAmPer Mulero, Miguel Ángel (ed.), Tiempos de investigación, JAECSIC, cien años de ciencia en España, Madrid, CSIC, 2007.

Rey PASTOR, Julio, «Esteban Terradas, su vida y su obra», en Real Academia de Ciencias Exactas, Físicas y Naturales, Discursos pronunciados en la sesión necrológica en honor del Excmo. Sr. D. Esteban Terradas e Illa el día 24 de enero de 1951, Madrid, 1951, pp. 35-64.

Roca Rosell, Antoni, «De la regeneración a la involución: Terradas y Rey Pastor, 35 años de amistad científica», en ESPAÑol GonZÁLEZ, Luis (coord.), Estudios sobre Julio Rey Pastor (1888-1962), Logroño, Instituto de Estudios Riojanos, 1990, pp. 71-104.

Romero de Pablos, Ana, «Mujeres científicas en la dictadura de Franco. Trayectorias investigadoras de Piedad de la Cierva y María Aránzazu Vigón», ARENAL, 24:2; julio-diciembre, 2017, pp. 319-348.

ROMERo De PABlos, Ana, «Historia de una biblioteca atómica», en CAMPRUBí, Lino; RoQué, Xavier; Saez de AdAna, Francisco (eds.), De la Guerra Fría 
Ciencia de papel. Privilegios y autoridad en España durante el primer franquismo (1939-1959)

al calentamiento global. Estados Unidos, España y el nuevo orden científico mundial, Madrid, Los libros de la Catarata, 2018, pp. 63-84.

Ruiz BAUTISTA, Eduardo, Los señores del libro: propagandistas, censores y bibliotecarios en el primer franquismo (1939-1945), Gijón, Trea, 2005.

SÁnchez Ron, José Manuel (coord.), La Junta para Ampliación de Estudios e Investigaciones científicas 80 años después (Vol. I/II), Madrid, CSIC, 1988.

SÁnchez Ron, José Manuel (comp.), En torno a la historia del CSIC, Arbor, Vol. 160., N. ${ }^{\circ}$ 631-632, 1998.

SANTESMASEs, María Jesús; MuÑoz, Emilio, Los primeros años del Consejo Superior de Investigaciones Científicas. Una introducción a la política científica del régimen franquista, Madrid, Documento de Trabajo, 1993.

Santesmases, María Jesús, Alberto Sols, Alicante, Ayuntamiento de Sax, 1998.

SAntesmases, María Jesús, Mujeres científicas en España (1940-1970) Profesionalización y modernización social, Madrid, Instituto de la Mujer, 2000.

SAnTESmases, María Jesús, «Viajes y memoria: las ciencias en España antes y después de la guerra civil», Asclepio, vol. LIX, n. ${ }^{\circ}$ 2, julio-diciembre, 2007, pp. 213-230.

SANTESMASES, María Jesús, «Conocimiento y autoridad: comentarios sobre la física en la dictadura de Franco», en HERRAN, Nestor y RoQué, Xavier (eds.), La física en la dictadura. Físicos, cultura y poder en España 1939. 1975, Bellaterra, Universitat Autónoma de Barcelona, 2012, pp. 295-310.

SANTESMASES, María Jesús, «Género, afición y mérito: una biografía de Sara Borrell Ruiz», ARENAL, 24:2; julio-diciembre, 2017, pp. 287-318.

SECORD, James A., Victorian Sensation. The extraordinary publication, reception and Secret Authorship of Vestiges of the Natural History of Creation, Chicago, The University of Chicago Press, 2000.

SOLER FERrÁn, Pablo, «La obra científica de Ramón Ortiz Fornaguera (19161974): un capítulo de la física matemática, teórica y nuclear de la dictadura franquista», Actes d'història de la ciencia $i$ de la técnica, Vol. 8, 2015, pp. 9-55.

Thompson, John B, Merchants of Culture. The Publishing Business in the Twenty-First Century, Cambridge, Polity, 2012.

\section{Datos del autor}

Fernando García Naharro es investigador postdoctoral en el Romanisches Seminar (Institut für Sprache, Literatur und Medien, Europa-Universität Flensburg, Alemania) y actualmente trabaja en el proyecto de investigación financiado por el BMBF "Antisemitismus im europäischen Schulunterricht (AIES)». Licenciado en Historia y en Antropología Social y Cultural y doctor en Historia Contemporánea por la Universidad Complutense de Madrid, sus áreas de interés 
incluyen la Historia del Libro y los Medios de Comunicación, la Historia de la Edición y la Lectura, los Estudios de Ciencia y Tecnología y los Estudios Culturales. Durante su formación ha realizado estancias de investigación en Inglaterra (University of Leeds), California (University of California Davis) y Cataluña (Universitat Autònoma de Barcelona). 